\title{
Stochastic sizing of isolated rural mini-grids, including effects of fuel procurement and operational strategies
}

\author{
Davide Fioriti ${ }^{\mathrm{a}, *}$, Romano Giglioli ${ }^{\mathrm{a}}$, Davide Poli ${ }^{\mathrm{a}}$, Giovanni Lutzemberger ${ }^{\mathrm{a}}$, \\ Andrea Micangeli ${ }^{\mathrm{b}}$, Riccardo Del Citto ${ }^{\mathrm{b}}$, Ignacio Perez-Arriaga ${ }^{\mathrm{c}}$, \\ Pablo Duenas-Martinez ${ }^{\mathrm{C}}$ \\ ${ }^{a}$ DESTEC, University of Pisa, Largo Lucio Lazzarino, 56122 Pisa, Italy \\ ${ }^{b}$ DIMA, University of Rome "Sapienza", Via Eudossiana 18, 00184 Roma, Italy \\ ${ }^{c}$ MIT Energy Initiative, Massachusetts Institute of Technology, \\ 77 Massachusetts Avenue, 02139 Cambridge (MA), United States
}

\begin{abstract}
The governments of developing countries struggle to guarantee the universal access to electricity on their territory and 1.2 billion people are still without any service, especially in remote areas. Hybrid mini-grids can be an effective solution since they exploit local renewable resources integrated with energy storage devices, reduce the use of fuel generators, and defer the construction of long and expensive grids until the growth of demand makes it profitable. Off-grid mini-grids are typically operated with simple load-following dispatching strategies, but predictive approaches can provide better performances, although at the expense of additional computational requirements. This paper investigates the benefits of using rolling-horizon dispatching strategies during the mini-grid design stage, also comparing how the optimal size of components is affected by several technical and economical parameters. Moreover, we propose the use of a stochastic sizing procedure that captures the uncertainties related to the load, to the renewable generation, and to the time required for the fuel procurement and delivery. A case study with real load data collected from an existing mini-grid placed in Habaswein, Kenya, is presented and discussed. The optimal sizing of some
\end{abstract}

\footnotetext{
*Corresponding author

Email addresses: davide.fioriti@ing.unipi.it (Davide Fioriti), andrea.micangeli@uniroma1.it (Andrea Micangeli), ipa@mit.edu (Ignacio Perez-Arriaga)
} 
components turns out to be almost unaffected by the operational strategies, so their preliminary design can be simplified to avoid time-consuming simulations. Conversely, the optimal sizing of the diesel generator and of its fuel tank is strongly related to both the local economic parameters and the operational strategy of the mini-grid, which must be properly simulated.

Keywords: Mini-grid, Isolated system, Fuel management, Monte Carlo, Optimization, Rolling-horizon dispatching

\section{Nomenclature}

$N P C$ Net Present Cost of the mini-grid project

$C A P E X$ Capital expenditures

$O P E X$ Operational expenditures

$S$ Number of Monte Carlo scenarios

$N_{L}$ Lifetime of the project in years

$T_{r}$ Time resolution

$T_{h}$ Period of time between two consecutive redispatching procedure

$T_{o}$ Time horizon of the MILP optimization

$C_{F, t}$ Fuel cost $(\$)$

$C_{C, t}$ Cost of load curtailment $(\$)$

$C_{M, t}$ Maintenance cost $(\$)$

$C_{R C, t}$ Cost of RES curtailment (\$)

$C_{S S}$ Cost of the overuse of the battery $(\$)$

$F_{D, t}$ Fuel consumption (l)

$P_{C, t}, P_{R C, t}$ Curtailment of the load and renewable energy sources $(\mathrm{kW})$

$P_{D, t}, z_{D, t}$ Diesel generator power $(\mathrm{kW})$ and its status (1: running, 0: off)

$P_{i n v, t}, P_{B, t}$ Inverter and battery power $(\mathrm{kW})$

$P_{L, t}$ Load $(\mathrm{kW})$

$P_{P V A v, t}$ Available renewable production $(\mathrm{kW})$

$P_{D}^{\max }, P_{D}^{\min }$ Maximum and minimum diesel power $(\mathrm{kW})$

$V_{F, t}$ Fuel available in the tank (1)

$E_{B, t}$ Energy available in the battery $(\mathrm{kWh})$

$E_{B}^{\max }, E_{B}^{\min }$ Maximum and minimum energy battery level $(\mathrm{kWh})$

$P_{B+}^{\max }, P_{B-}^{\max }$ Maximum and minimum battery power $(\mathrm{kW})$

$P_{i n v+}^{\max }, P_{\text {inv- }}^{\max }$ Maximum and minimum inverter power $(\mathrm{kW})$

$c_{D S, i}, c_{D I, i}$ Piecewise linearization of the fuel consumption (offset and slope)

$c_{s s}$ Specific cost of the battery overuse $(\$ / \mathrm{kWh})$

$\eta_{I}, \eta_{B}$ Efficiency of the inverter and battery (-) 


\section{Introduction}

Nowadays, while industrialized countries have completed their electrification process, governments of developing countries are still struggling to provide a reliable access to electricity to around 2.2 billion people worldwide $[1,2]$, of which 1.2 billion lacks any service [1]. Mini-grids are widely considered one of the most effective solutions to supply areas far from the grid or with weak infrastructure, since they can defer the construction of long and expensive grids until the growth of demand makes it profitable. In this context, planning tools are really helpful to identify the cheapest electrification option even at country scale [3, 4].

International and national efforts are in particular promoting hybrid solutions that include renewable sources and energy storage $[2,5,6]$, not only to comply with the COP21 guidelines and reduce local pollution, but also to reduce the project costs, the renewable sources being already competitive with fossil fuel technologies in many contexts [5]. Business and technical risks in mini-grid investments are still significant [7], although the huge potential market of USD 200 billion/year [5] is attracting not only non-governmental organizations, research institutions, and public investors, but also private companies.

Predictive operational strategies of resources can improve the efficient use of fuel-fired generators and reduce load shedding with respect to the traditional load-following procedures $[8,9]$. In addition, stochastic sizing methodologies are very promising tools in order to properly cope with the uncertainties related to load, renewable sources and fuel procurement, assuring a robust and reliable probabilistic design of the mini-grid, including the fuel tank capacity.

For these reasons, this paper proposes and analyzes a stochastic sizing technique that combines a probabilistic approach, an optimization tool and the simulation of a predictive operational strategy of resources including their intra-daily redispatching. The benefits obtained with respect to traditional load-following procedures are investigated and numerically discussed for an isolated system, also performing a wide sensitivity analysis of results when techno-economic local constraints and parameters change. Moreover, the

optimal sizing of the fuel tank is explicitly considered into the design of the mini-grid, turning out to be particularly dependent on the average and variance of the time required for fuel delivery. 


\section{Problem formulation}

\subsection{Uncertainties in sizing methodologies}

The literature regarding the optimal sizing of power systems under uncertainties is very rich, especially for hybrid systems with multiple energy sources, isolated or linked to the national grid [10-14]. Typical methodologies iteratively simulate the operation of the system, testing several combinations of the size of the components [8, 15-18] in order to find the design that minimizes total system costs, also including reliability aspects and other socio-economic indicators $[16,19]$.

Conventional commercial software usually optimizes only a single deterministic scenario of load and renewable generation [17], while the scientific literature is recently proposing several approaches to make decisions under uncertainty, which means taking into account many possible profiles of load and renewable generation. Authors in [20] applied for instance a chanceconstrained method to size and locate distributed energy sources, assuming that load flows can exceed the maximum capability of feeders according to a probability density function assessed by simulating multiple scenarios of load, distributed generation, and energy price. According to the approach suggested in [18], the best design of an isolated system can be obtained by deterministically optimizing the size of its component in several independent scenarios of load and renewable generation, previously drawn by a Monte Carlo procedure; the final best design is then obtained by analyzing the statistical properties of occurrence of the different scenarios. A similar approach is also proposed in [21], where the point estimate method is applied to an interconnected system, or in [22], in relation to an isolated system. Authors in [11] proposed a stochastic approach based on NSGA-II method to size a hybrid system able to meet the thermal demand under uncertainties in solar and wind production; the outcome is the Pareto front between the economic value of the energy-not-served and the investment costs. The study [17] details a robust approach by using multiple scenarios to size rural systems in developing countries, but robustness seems to lead to rather conservative results. Conversely, papers $[8,12,15,23]$ propose to approximate the stochastic objective function of the problem into a weighted sum of the deterministic objective functions of single scenarios. This allows estimating the operational costs, the maintenance fees, and the energy-not-served under different conditions of load or renewable production, by weighting the outcomes of the different scenarios with their probability to occur. More in detail, authors in 
$[8,15]$ firstly proposed to use this methodology to size also the fuel tank and the fuel logistics. The study in [15], based on a Particle Swarm Optimization (PSO) method to best select size scenarios to be investigated, was improved in [8], where the same authors included a rolling-horizon dispatching strategy of the batteries and of the diesel generator. The results were compared to the ones obtained by implementing a conventional load-following operating procedure, but the validity of this comparison were limited to a single techno-economic scenario, tuned for a possible mini-grid to be installed in Uganda. A wide sensitivity analysis is instead required to verify, generalize and enforce this comparison, moreover using realistic data collected from the field, as performed in the present paper. This enabled achieving additional results and fine-tuning design criteria, as discussed in the next sections.

The simulations carried out by the optimization techniques usually cover a time horizon long enough to capture the periodicities of both the load and renewable sources, which means at least an entire year [15, 17, 18, 22]. A horizon of multiple years enables evaluating the degradation of the components [24], but the longer the time span, the higher the necessary computational time $[8,25]$; thus, model simplifications are required, such as in [25] where the entire year was sampled in a few representative weeks. For yearly simulations, the available renewable production is often estimated by using Typical Meteorological Year (TMY) data [24] that incorporate the random behaviour of renewable generation [26]. Nevertheless, many authors [8, 15, 18, 22] proposed synthetic methods to draw realistic yearly profiles of the available renewable production [27-29]; synthetic approaches are useful for stochastic sizing, since enabling to analyze many realistic years, as in $[12,15,18,22]$.

\subsection{Fuel tank sizing}

According to our literature analysis, papers referring to "tank sizing" in mini-grids focus on sizing hydrogen [30] or water tanks [31], while the ones citing the fuel tanks only focus on large power plants, e.g. in [32]. The optimization of the size of the fuel tank is usually not included into the sizing procedure of the whole mini-grid, probably because of the implicit assumption that the operator always assures that the tank is being properly refilled. However, recent papers $[8,15]$ suggested that the distribution function of the time required for the fuel delivery has a certain influence on the optimal size of the fuel tank. This dependence is worth investigating, because of its importance for remote areas with poor logistic infrastructures, such as in many sites in Africa or in the Amazon. 


\subsection{The daily dispatching of energy resources}

Due to their simplicity and robustness, traditional operational strategies of existing isolated mini-grids are non-predictive, such as load following and cycle charging. Both procedures turn on the fuel generator when the battery reaches a minimum State-Of-Charge (SOC), but in the first strategy the fuel generator supplies only the load, leaving the battery at its SOC, while in the cycle-charging method the generator feeds also the battery until it reaches a preset SOC. This concept was firstly introduced in [33], then tested in many papers $[15,34]$ and implemented in commercial software [24]. When the mini-grid is interconnected to a wider power system, its operation must comply with the procedures set by the relevant utility or market operator [35]. Moreover, the simulations should include a proper model for the actual availability of the interconnection that could be rather unreliable in the rural areas of developing countries [25].

Predictive strategies are conversely based on the short-term forecasting of load and RES production, which enables dispatching in advance the batteries and the fuel generator, thus preventing and mitigating out-of-charge events and load curtailment; furthermore, optimal dispatching procedures enhance the efficiency of the working point of the fuel generator $[8,9,36]$, which in load-following procedures depends on the residual load to be met.

Advanced predictive methods perform a rolling-horizon approach, where the optimization of resources is repeated also intra-daily to cope with the load and RES forecasting errors, as well as with the contingencies occurred along the real time operation of the mini-grid $[8,9]$. Other stochastic methodologies embed the statistical properties of load and RES uncertainties [37], but their complexity and time requirement discourage their use for sizing purposes.

\section{The simulation of the mini-grid's operation}

The mini-grid's operation includes the possible short-term scheduling of energy resources, their real-time dispatching, as well as fuel procurement and maintenance issues, as discussed in this section for the typical scheme of Fig. 1, which is very common in rural areas of developing countries. Clustering mini-grids or expanding the main national grid could be valid alternatives to isolated systems, to be investigated case by case. In this paper, we assume that upstream planning tools like the Reference Electrification Model (REM) [4] have already identified the isolated mini-grid as the most promising topology for the site under analysis; this said, the proposed procedure provides 
more accurate criteria for refining the size of each component. In addition, the gained accuracy may help to better classify ex-ante if a mini-grid or grid expansion is the most economical solution for a specific set of consumers. This analysis is part of our future research guidelines.

Its also worth noticing that in mini-grids the short distances make singlebusbar models an acceptable simplification, especially when focusing on the optimal design of power sources and storage devices, of course provided that the considered load includes realistic distribution losses.

Load following and a novel predictive strategy were both simulated for comparison purposes, as the most representative of the possible dispatching methodologies, according to [33].

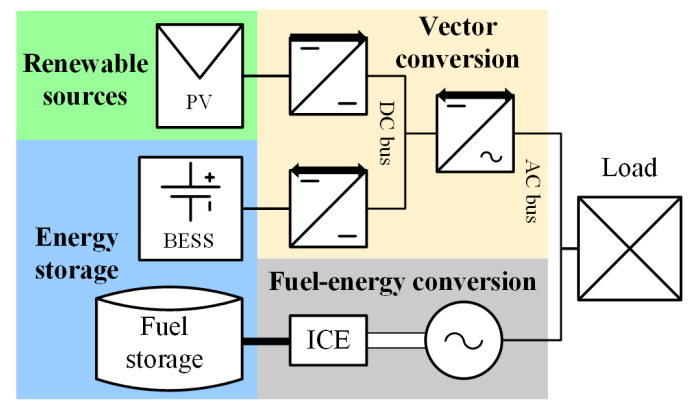

Figure 1: Typical design of a rural mini-grid.

\subsection{Load-following strategy (LFS)}

The load-following strategy, typically used in rural mini-grids, consists of priority-list rules that in real time first exploit the renewable sources, then the energy stored in the battery, and lastly the fuel. The battery balances the mismatch between load and the renewable production according to its power and energy limits. When the battery is fully charged, the extra renewable production is curtailed; conversely, when the minimum SOC is reached, the diesel generator is turned on to possibly avoid load curtailments, without charging the battery. This methodology requires no RES or load forecasting and has minimum computational complexity.

\subsection{Predictive strategy}

Predictive strategies are more complex than load-following or cycle-charging procedures, but their ability to dispatch the mini-grid anticipating future 
needs can reduce operational costs, the sizing of the backup fuel generator and the energy not served $[8,15]$. In fact, with predictive strategies the optimal operation of storage devices makes it possible, for instance, to recharge in advance the batteries based upon the upcoming prices (when a day-ahead market sets the hourly prices or a Time Of Use charge is applied), irradiation and load, by anticipating the start-up of the diesel generator.

\subsubsection{Rolling horizon scheduling of resources (RHS)}

In this paper, the rolling-horizon procedure depicted in Fig. 2 is proposed and discussed. Every $T_{h}$ hours, the load and the available renewable production of the following $T_{o}$ hours are forecasted and the corresponding use of batteries and diesel generator is rescheduled at minimum operational costs, using a mixed-integer linear programming (MILP) algorithm. The new scheduling is maintained for the following $T_{h}$ hours, up to the next iteration of the procedure. At this stage, operational expenses include not only fuel costs and the economic value of the non-served energy, but also the overuse cost of the battery. This last addend is an economic penalty considered when the energy stored in the battery at the end of the optimization period turns out to be below its initial SOC. Such a cost is estimated considering the additional costs that the overuse of the battery would cause in the next optimization period, in terms of additional load curtailment or supplementary diesel production.

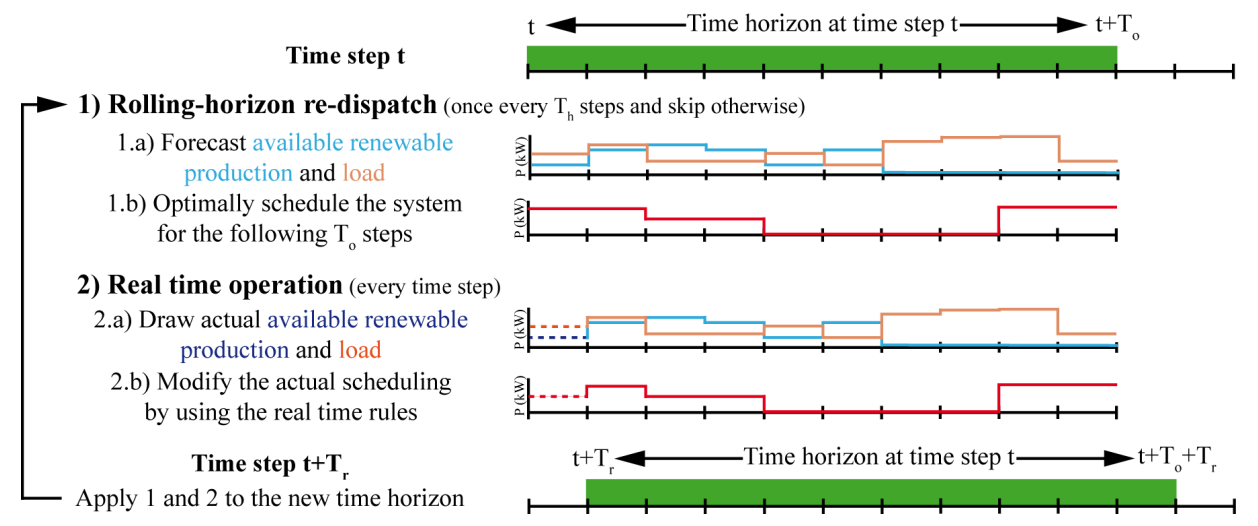

Figure 2: Rolling-horizon predictive strategy.

The objective of the proposed MILP procedure is to minimize operational costs, including fuel expenses $C_{F, t}$, the economic value of the load curtailment 
$C_{C, t}$, and the maintenance $C_{M, t}$, as in (1). The additional overuse cost $C_{S S}$ is applied if the final energy available in the battery $E_{B, 24}$ is lower than its initial value $E_{B, 0}$. The value of $c_{S S}$ has been set to the specific cost of the diesel generator when running at its maximum efficiency.

Eq. (1a) expresses the objective function to be minimized. Equations from (1c) to (1f) relate specific and total costs of unserved energy, battery overuse, maintenance, fuel, and renewable curtailment, respectively.

$$
\begin{array}{cr}
\forall t & \min \sum_{t=1}^{T} C_{F, t}+C_{C, t}+C_{M, t}+C_{R C, t}+C_{S S} \\
& C_{S S}=\left\{\begin{array}{cc}
0 & \text { if } E_{B, 24}>E_{B, 0} \\
c_{S S}\left(E_{B, 24}-E_{B, 0}\right) & \text { otherwise }
\end{array}\right. \\
\forall t & C_{C, t}=c_{C} P_{C, t} \\
\forall t & C_{M, t}=c_{M} z_{D, t} \\
\forall t & C_{F, t}=c_{F} F_{D, t} \\
\forall t & C_{R C, t}=c_{R, t} P_{R C, t}
\end{array}
$$

The power balance in the $\mathrm{AC}$ and $\mathrm{DC}$ bus is guaranteed by equations (2a) and (2b), respectively:

$$
\begin{array}{cc}
\forall t & P_{D, t}+P_{i n v+, t}-P_{i n v-, t}=P_{L, t}-P_{C, t} \\
\forall t & P_{B+, t}-P_{B-, t}+P_{i n v+, t} \eta_{I}-\frac{P_{i n v-, t}}{\eta_{I}}+P_{P V A v, t}=P_{R C, t}
\end{array}
$$

The equation group (3) details the diesel constraints, including the fuel consumption (3b) and tank level (3c).

$$
\begin{array}{rc}
\forall t & P_{D, \min } z_{D, t} \leq P_{D, t} \leq P_{D}^{\max } z_{D, t} \\
\forall t, i & F_{D, t} \geq c_{D S, i} P_{D, t}+c_{D I, i}-\left(1-z_{D, t}\right) M \\
\forall t & V_{F, t}=V_{F, 0}-\sum_{t^{\prime}=1}^{t} F_{D, t^{\prime}} \geq 0
\end{array}
$$

The power and energy constraints of the inverter, the battery, and the battery converter are expressed by the following equations (4).

$$
\begin{array}{cc}
\forall t & 0 \leq P_{i n v+, t} \leq z_{I, t} P_{i n v+}^{\max } \\
\forall t & 0 \leq P_{i n v-, t} \leq\left(1-z_{I, t}\right) P_{i n v-}^{\max } \\
\forall t & E_{B}^{\max } \leq E_{B, t} \leq E_{B}^{\max } \\
\forall t & E_{B, t}=E_{B, 0}+\sum_{\widehat{t}=1}^{t}-\frac{P_{B+, \widehat{t}}}{\eta_{I}}+\eta_{B} P_{B-, \widehat{t}} \\
\forall t & 0 \leq P_{B+, t} \leq z_{B, t} P_{B+}^{\max } \\
\forall t & 0 \leq P_{B-, t} \leq\left(1-z_{B, t}\right) P_{B-}^{\max }
\end{array}
$$


Finally, the constraints relevant to the load shedding $P_{C, t}$ and to the curtailment of renewable sources $P_{R C, t}$ are detailed in (5).

$$
\begin{gathered}
\forall t \quad 0 \leq P_{P V, t} \leq P_{P V A v, t} \\
\forall t \quad 0 \leq P_{C, t} \leq P_{L, t}
\end{gathered}
$$

\subsubsection{Real-time operation}

Due to the intrinsic forecasting errors, a real-time controller is then required to balance the mismatch between the actual power profiles and their forecasts, basically applying a load-following procedure to power deviations. The following rules apply at each time step [15].

1. The diesel is dispatched as scheduled by the MILP, renewable sources are exploited at most, and the battery balances the power mismatch until it reaches its maximum capacity limits. Renewable curtailment occurs if the energy cannot be stored nor supplied to the load;

2. When the battery is out of charge or reaches its capacity limit while feeding the load, and the actual demand is higher than the dispatched production, then:

(a) if the diesel generator was committed to produce by the MILP, its scheduling is increased until required;

(b) if the diesel generator was off according to the MILP, the economic value of curtailing the extra load is compared to the cost of turning on the generator, and the cheaper option is chosen.

3. When the battery is full or reaches its power limit while being charged, the actual production is higher than the load, and the mismatch is balanced according to the following merit-order list: first, the diesel production is reduced even to shut-down; then, the renewable production is curtailed.

Any variable under simulation is discretized into time steps of $T_{r}$ hours (usually $0.25 \mathrm{~h}, 0.5 \mathrm{~h}$ or $1 \mathrm{~h}$ ) that must be short enough to capture the system dynamics. The choice of $T_{r}$ and $T_{o}$ is strictly related to the load and renewable power profiles: $T_{r}$ captures their fast dynamics, while $T_{o}$ must embrace the longer. Moreover, short values of the period $T_{h}$ between two consecutive redispatches allow to frequently re-optimize the system and compensate for the unavoidable forecasting errors. However, frequent redispatchings cause a sharp increase in computational time, especially with low values of the time resolution $T_{r}$ and long time horizons $T_{o}$. Since the values of $T_{r}, T_{h}$ 
and $T_{o}$ influence also the accuracy of results, their optimal choice requires a compromise between optimality and computational requirements [8].

\subsection{Fuel procurement}

\subsubsection{Route strategies}

The on-site price of fuel is significantly increased by the cost of transportation, usually performed with tank trucks. Transportation costs depend on the quality of roads and on the route taken to reach the mini-grid, and less on the quantity of the transported fuel. Therefore, the delivery of larger fuel volumes is attractive to decrease the per unit impact of fixed costs. Two possible strategies to refill a cluster of mini-grids are described below.

- Individual strategy: the fuel delivery is limited to a single mini-grid.

- Collective strategy: the fuel delivery is aimed at refilling a cluster of mini-grids, so the specific fuel price is reduced since transportation fixed costs are shared among multiple users. Moreover, this strategy usually involves the use of larger trucks, whose impact on specific fuel cost decreases because of the clear economies of scale.

\subsubsection{Model of the fuel delivery timing}

The tank truck logistics strongly influences the delivery time $t_{D}$ whose general mathematical model is expressed by Eq. (6), where $t_{P}$ and $t_{T}$ are the deterministic minimum time required for managing the procurement process and the transport, respectively; the term $p d f$ is the probability density function (PDF) that models the stochastic components of the availability of the truck, of the management structure and of the transport time. More details about the term $p d f$ are provided in the case study.

$$
t_{D}(h)=t_{P}+t_{T}+p d f
$$

\section{The sizing procedure}

As previously mentioned, the mini-grid is sized by searching the set of components whose operation is cheapest, also taking into account the investment costs and the economic value of load curtailment. In more detail, the stochastic procedure proposed to size the mini-grid is composed of the two nested loops depicted in Fig. 3. In the outer loop, an optimization strategy, such as Particle Swarm Optimization (PSO) or Imperialist Competitive 
Algorithm (ICA) [15, 38], iteratively selects possible configurations of the size of the components. Their operation is then simulated in the inner loop, according to the procedures discussed in the previous section, for $S$ different Monte Carlo annual scenarios of load and the available renewable production. Finally, the procedure calculates the objective function associated to the size configuration under test and, if one of the termination criteria is reached, the iterations stop and the optimal size scenario is identified.

This methodology can be applied to both greenfield projects and existing mini-grids under revamping, provided that the assessment of the load and of its probability density function is performed accordingly. Demand assessment is out of the scope of this paper and the expected load profile, together with the distribution function of the relevant short-term forecast errors, is considered as an input of our procedure; previous papers of the same authors $[8,15]$ and a lot of scientific literature have dealt with this topic. In this regard, the novelty of the approach proposed in this paper is that a probabilistic model of load and renewables is combined with optimal dispatching procedures and an external loop with sizing purposes. Monte Carlo scenarios are in fact used to cope with the day-ahead uncertainty of the load and of the renewable generation, for purposes of predictive daily dispatching. The uncertainty correspondent to the long-term evolution of the load is not simulated in this paper; the average power profiles assumed as an input of our procedure must be in fact considered as representative of a mid-term perspective that is used to approximate the multi-annual behavior of the mini-grid, as in $[8,17,18,22,25]$. Nevertheless, its worth noticing that the long-term uncertainty of the load, be it deterministically or probabilistically treated, could be also addressed in our tool by considering different expected load profiles, to be varied or drawn in a long-term perspective, rather than just considering the daily Monte Carlo deviations with respect to a single predefined yearly profile.

The proposed tool has already been validated in the past with respect to HOMER when adopting a load-following strategy [39]; the comparison between the load-following strategy and the predictive dispatching was instead discussed in [8], but only for a single configuration of operating costs and fuel procurement, besides on a theoretical case study not supported by real electrical measurements. In this paper, we improved the simulation tool and enforced the previous comparison through a sensitivity analysis of operating costs and of the time required for fuel procurement; in addition, the expected load and generation profile, as well as the probability density functions of the 
relevant short-term forecasting errors, were tuned on real measurements collected in an existing mini-grid under refurbishment.

\subsection{Objective function of the sizing procedure}

The objective function to be minimized in the sizing procedure is the expected Net Present Cost (NPC) of the system during its lifetime $\left(N_{L}\right)$, expressed by Eq. (7). In Eq. (7), while CAPEX are deterministic since they are fixed once the size scenario under examination is established, OPEX are a stochastic function related to the power profiles of load and renewable production, including their uncertainty. By using the Sample Approximating Algorithm [40], the resulting OPEX in (8) is the average value of the operational costs obtained in the $S$ Monte Carlo scenarios.

$$
\begin{gathered}
N P C=\sum_{y=0}^{N_{L}} \frac{C A P E X_{y}+O P E X_{y}}{(1+i)^{y}} \\
O P E X_{y}=\sum_{s=1}^{S} \frac{O P E X_{y_{s}}}{S}
\end{gathered}
$$

CAPEX includes the investment costs of the diesel-fired generator, batteries, converters, renewable generators and fuel tank; OPEX includes fuel costs, maintenance fees and the economic value of the energy not served.

\subsection{Outer loop: optimization}

After a first initialization, the outer loop iteratively selects the new size of components to be analyzed, using a predefined algorithm such as PSO or ICA $[15,38]$. Once the configuration in the present iteration is selected, the algorithm evaluates the corresponding CAPEX and starts the simulation of the mini-grid operation, as detailed in the inner loop. As a compromise between the optimality of results and the computational complexity, we used a PSO algorithm with the following termination criteria: number of iterations (200), maximum number of stall iterations (12), and tolerance $\left(10^{-3}\right)$.

\subsection{Inner loop: stochastic simulation}

One of the main novelties of this paper is within the inner loop, where the stochastic nature of the load, of the available renewable production and of the time required for fuel delivery are captured by simulating the system 


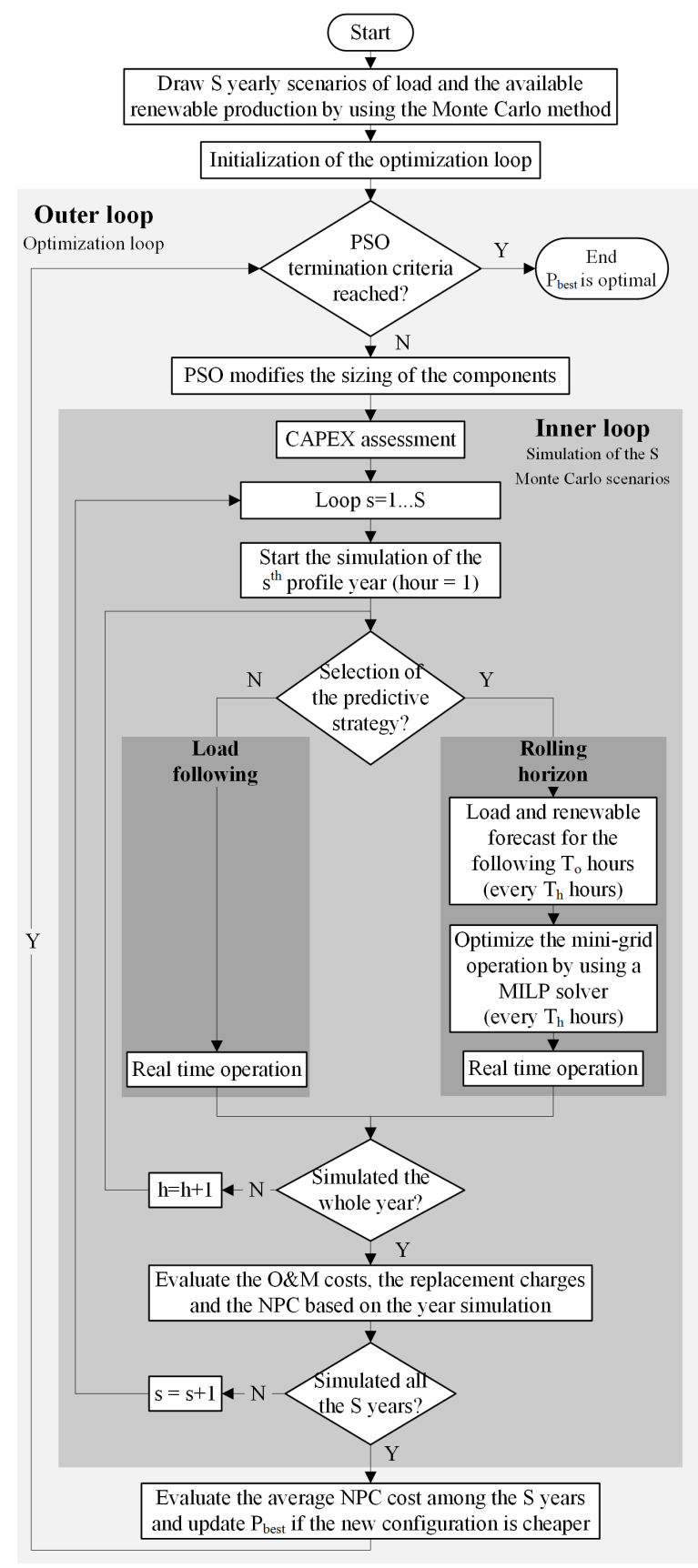

Figure 3: Scheduling and operation of resources. 
operation in $S$ yearly possible time profiles of stochastic quantities, drawn by a sequential Monte Carlo technique [41, 42].

As depicted in Fig. 3, this procedure is valid for both the load-following and rolling-horizon strategies. However, when the predictive strategy is selected, the procedure starts by determining the forecast of the load and of the available renewable production of the following $T_{h}$ hours, by using two Gaussian PDFs that model forecasting errors. After that, the MILP algorithm calculates the optimal scheduling of the mini-grid and finally the real time rules balance the on-line operation of the system. In more detail, the standard deviations of the two Gaussian distribution functions are assumed to linearly increase from the $5 \%$ at the first to $15 \%$ at the last time step of the rolling-horizon window. The time required for fuel delivery is instead drawn according a Weibull PDF, according to Eq. (6).

Conversely, when the load-following strategy is adopted, only the real time balancing rules are applied, without any forecasting activity.

\section{Description of the case study}

The proposed methodology is applied to a mini-grid to be installed in Habaswein, Wajir County, Kenya (1.0N, 39.29E). Population is about 10,000 people, mainly engaged in the agriculture sector [43]. The proximity to the Equator confirms the validity of the mini-grid configuration depicted in Fig. 1, composed by a photovoltaic plant, a lithium battery storage, an inverter, a diesel generator, and a fuel tank.

\subsection{Simulation parameters}

We performed the simulations with hourly time steps $\left(T_{r}=1 \mathrm{~h}\right)$, using both load-following and predictive strategy, for comparison purposes. The predictive strategy re-optimizes the system dispatching every 6 hours $\left(T_{r}=\right.$ $6 h)$, as a compromise between computational requirements and the frequency of optimal rescheduling of resources. Each MILP procedure minimizes the operational costs expected over the following 24 hours $\left(T_{o}=24 h\right)$. We selected this time window because the daily periodicity best describes the patterns of local activities and the available renewable production.

The simulations were performed using 5 Monte Carlo scenarios and assuming the lifetime of the project is 15 years [44, 45]. This lifespan is a challenging target that requires good maintenance and quality products, es-

pecially for inverters. However, nowadays several manufacturers propose base 
warranties of 10 years, extendable up to 15, 20 or even 25 years. The significant maintenance costs considered in our case study are consistent with the purposes of good preservation of components, especially the inverter, whose cooling components must be kept well clean; yearly maintenance includes filters substitution and proper internal cleaning. CAPEX reflect both warrant extensions and the need to change the fans of the inverter after 10 years of operation.

\subsection{Load profile}

The load profiles used for simulating the real time operation of the minigrid are based on measures collected in a nearby similar minigrid for the entire year 2014, with a granularity of 30 minutes. In order to represent the stochastic nature of our analysis, the $\mathrm{S}$ yearly profiles drawn by the Monte Carlo procedure are obtained by adding to the historical pattern an additional Gaussian noise, having null average and a standard deviation equal to $20 \%$ of the actual demand, measured in 2014 in the analyzed time step. As discussed at the beginning of Section 4, this yearly load profile is assumed to represent the mid-term stress of the mini-grid.

A sensitivity analysis on the cost of load curtailment was performed, assuming possible values of $0.5 \$ / \mathrm{kWh}$ and $1 \$ / \mathrm{kWh}$.

\subsection{Available photovoltaic production}

The irradiance reaching the photovoltaic modules and the local ambient temperature are the main drivers of the available photovoltaic production [46]. The hourly profiles of incident irradiance used for simulating the real time operation of the mini-grid were established using the Graham algorithm $[28,29]$ combined to the HDKR technique [46]. The methodology is based on ARMA models that were tuned on the data available from the Kenyan meteorological station of Kitale. In order to foster the use of the renewable source at the beginning of the time horizon optimized by the MILP procedure, where its forecast is more accurate, the cost of PV curtailment decreases from $0.01 \$ / \mathrm{kWh}$ in the $1^{\text {st }}$ hour down to $0 \$ / \mathrm{kWh}$ at the $24^{t h}$.

\subsection{Fuel cost and procurement}

We assumed that a new fuel delivery is requested when the remaining fuel quantity reaches a fixed threshold (20\%). Then, a tank truck transports a fixed fuel volume ( $80 \%$ of the mini-grid tank) to the mini-grid. The time elapsed between the fuel request and its arrival is modeled using a Weibull 
PDF in (6), calibrated according to Table 1. A sensitivity analysis on the allinclusive cost of fuel was also performed, assuming possible values of $0.8 \$ / 1$, $1.2 \$ / 1$, and $1.6 \$ / 1$.

\begin{tabular}{l||c|c|c} 
Case & $\begin{array}{c}t_{P}+t_{T} \\
\text { (days) }\end{array}$ & $\begin{array}{c}50^{t h} \text { percentile } \\
\text { (days) }\end{array}$ & $\begin{array}{c}90^{t h} \text { percentile } \\
\text { (days) }\end{array}$ \\
\hline \hline $\mathrm{A}$ & 1 & 1.5 & 3 \\
$\mathrm{~B}$ & 2 & 3 & 6 \\
\hline
\end{tabular}

Table 1: Delivery time.

\subsection{Investment costs and efficiencies}

The investment costs of the main components were estimated using Eq. (9), where $P_{x}$ is the size under investigation, $\alpha$ the reference cost of the component with size $P_{0}$, and $\beta$ the exponent that depends on the economies of scale and of volume of the technology. The parameters of the equation, calibrated within the size range under investigation, are reported in Table 2, along with the expected maintenance costs; economic parameters, especially in terms of economies of scale, were tuned from the standpoint of an operator that is supposed to install and operate several mini-grids. The cost of the balance of plant (BoP) is included only for the share that dipends on the size of the component; the remaining part can be disregarded since not affecting the optimization.

$$
C(x)=\alpha\left(\frac{P_{x}}{P_{0}}\right)^{\beta}
$$

The efficiency of the components is $99 \%$ for the battery converter, $96 \%$ for the inverter, and $96 \%$ (roundtrip) for the battery pack, which corresponds altogether to a realistic $87 \%$ AC-AC roundtrip efficiency. The efficiency of the diesel generator increases roughly linearly from $11 \%$ (at technical minimum, i.e. $10 \%$ of rated power) up to $33 \%$ (at nominal output) [8].

\section{Results and discussion}

Fig. 4, Fig. 5, Table 3, and Table 4 summarize the results of the optimization cases carried out with MATLAB, according to the two described strategies (LFS and RHS), to the sensitivity analysis proposed in Section 5 and to the procedure described in Fig. 3. The 24 cases of the sensitivity 


\begin{tabular}{l||c|c|c|c} 
Asset & $P_{0}$ and $\mathrm{UM}^{*}$ & $\begin{array}{c}\alpha \\
\left(\$ / \mathrm{UM}^{*}\right)\end{array}$ & $\begin{array}{c}\beta \\
(-)\end{array}$ & $\begin{array}{c}\text { Maintenance } \\
(\$ / \mathrm{UM} / \mathrm{y})\end{array}$ \\
\hline \hline PV & $1 \mathrm{~kW}$ & 800 & 1 & 16 \\
Battery & $1 \mathrm{kWh}$ & 350 & 1 & 3 \\
Bat. conv. & $1 \mathrm{~kW}$ & 1258 & 0.5 & 2 \\
Inverter & $1 \mathrm{~kW}$ & 1887 & 0.5 & 2 \\
Fuel gen. & $1 \mathrm{~kW}$ & 1013 & 0.8 & $0.05 \$ / \mathrm{kW} / \mathrm{h}$ \\
Fuel tank & $1 \mathrm{liter}$ & 52.2 & 0.45 & 0.15 \\
\hline
\end{tabular}

*unit of measurement.

Table 2: Cost parameters of the main components, calibrated with $[8,15,47-51]$.

analysis required roughly one month $24 / 7$ on a 12 -core PC with 16GB RAM, of which few hours for each LFS case and some days for each RHS case; these are requirements still acceptable for sizing purposes in comparison to the time required for the feasibility phase of mini-grid projects.

Fig. 4 reports the NPC of the mini-grid for different values of the cost of the energy not served, fuel price and implemented strategy, averaged for scenarios A and B of time required for fuel delivery; small error bars in the figure show minimum and maximum obtained values. The effectiveness of the predictive strategy is clear: the NPC obtained with the RHS approach is always lower or equal to the one resulting from load following. In fact, when the optimal configuration of the mini-grid includes a diesel generator, RHS is always cheaper than LFS. Conversely, when installing a diesel generator is not cost-effective, which occurs when the economic value of the non-served energy is minimum and the fuel price is maximum, the RHS strategy cannot optimize the operation of the backup source, therefore the results of RHS are very related to the ones of LFS.

Fig. 4 also shows that the higher the cost of load curtailment, or the lower the fuel price, the higher the benefits of using the RHS. In the extreme case, using RHS the NPC decreases by $3 \%$. This cost reduction is both related to the reduction of CAPEX and to the increased continuity of supply. In fact, RHS reduces load curtailment by $60-65 \%$, on average. The NPC significantly depends on fuel price and on the cost of the non-served energy; the impact of the time required for fuel delivery is instead negligible.

Fig. 5 shows the optimal size of the PV plant, of the converters, and of the storage device, for different values of the cost of load curtailment, of the 


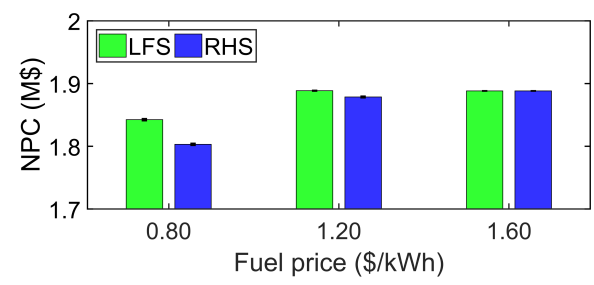

a) Load curtailment $0.5 \$ / \mathrm{kWh}$.

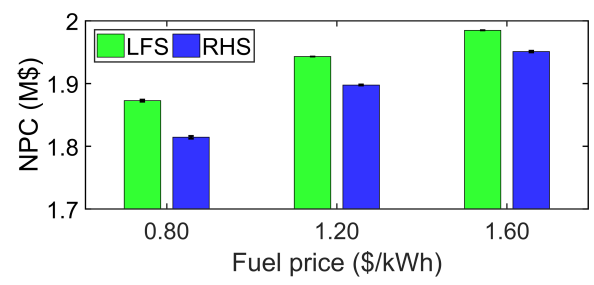

b) Load curtailment $1.0 \$ / \mathrm{kWh}$.

Figure 4: NPC of the mini-grid for different dispatching strategies, cost of load curtailment and fuel price; values are averaged on scenarios A and B of time required for fuel delivery.

fuel price and with the two possible dispatching strategies. The results are expressed in terms of percent difference between each optimal solution and a base case that is the optimal design obtained adopting the load following strategy, assuming a fuel cost of $0.8 \$ / 1$, and penalizing load curtailment with $0.5 \$ / \mathrm{kWh}$ (806-kWp PV plant, a $412-\mathrm{kW}$ battery converter, a $234-\mathrm{kW}$ inverter, and 2.25-MWh storage device). The values are averaged for scenarios $\mathrm{A}$ and $\mathrm{B}$ of time required for fuel delivery. As in Fig. 4, small error bars indicate minimum and maximum obtained values, showing that the dependence of sizes on fuel procurement is negligible. Focusing on dispatching strategies, with RHS the optimal size of these components is usually $2-8 \%$ lower than applying LFS (with very few exceptions on power electronics, whose size differs by $10-16 \%$ in a couple of scenarios). Introducing RHS therefore allows only a relatively low reduction of sizes, which suggests that the preliminary design of the PV plant, of the battery, and of the converters could be effectively assessed using the simple load following strategy, more than 500 times faster than the predictive one. Fig. 5 also shows that by doubling the fuel price or the cost of load curtailment the optimal sizes of the PV plant and of the battery differ by no more than $11 \%$ from their average value, $19 \%$ in case of converters.

Conversely, Table 3 shows that the optimal size of the diesel generator is strictly dependent not only on the adopted dispatching strategy, but also on the fuel price and on the cost of load curtailment, while remaining only slightly related to the fuel delivery time (A/B). According to [8, 15], Table 3 confirms for a wider sensitivity range that the higher the fuel price, or the lower the cost of non-served energy, the lower the optimal size of the diesel generator. It's worth noticing that with RHS the size of the generator is approximately halved with respect to LFS, because the use of RHS maximizes 
Load curtailment $0.5 \$ / \mathrm{kWh}$

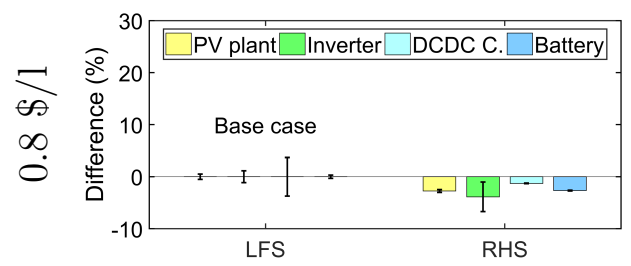

A1

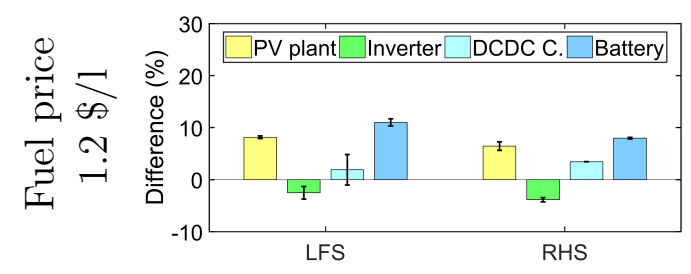

B1

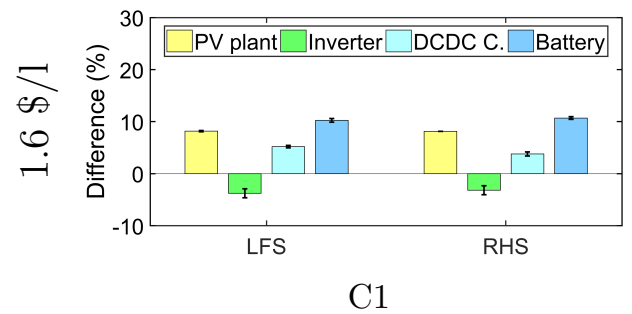

Load curtailment $1 \$ / \mathrm{kWh}$

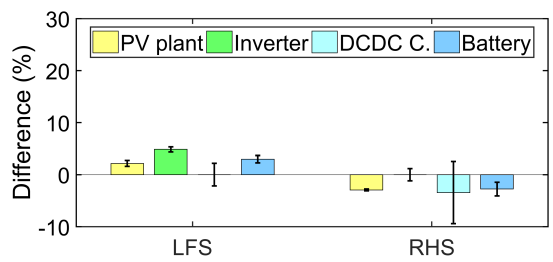

A2

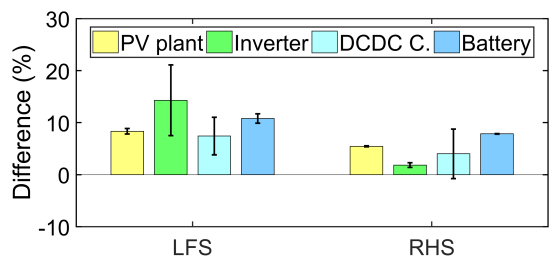

B2

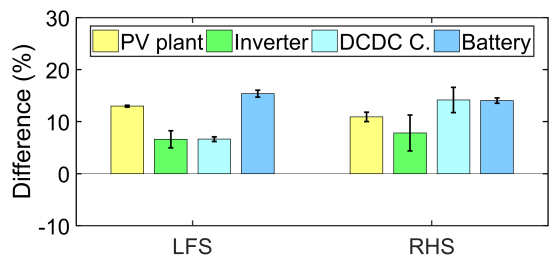

$\mathrm{C} 2$

Figure 5: Optimal size of the photovoltaic plant, the inverter, the DC/DC converter and the battery, for different dispatching strategies, cost of load curtailment and fuel price; each size is expressed in terms of percent difference with respect to the optimal solution obtained by applying LFS to case A1.

the coordination of the components also at the operational level, enabling to foresee the unbalances of the system and to prevent the batteries from being discharged. Simulations also show that at the same time RHS increases the energy produced by the diesel generator, by $28-45 \%$. This means that the rolling horizon redispatching procedure strongly increases the frequency of use of the diesel generator, while reducing its required capacity. The growth of diesel production is related to the reduction of load curtailment, in all scenarios. Since RHS boosts the usage factor of the diesel generator, also the efficiency of its working point improves. This is confirmed by the fact that implementing RHS the fuel consumption increases slightly less (from 3 to $6 \%$ ) than the produced electricity. The dependency of the diesel generator size on the timing of fuel delivery (case A and B) is always less then $5 \%$, except when 
adopting RHS with very high values of fuel price and cost of load curtailment. Moreover, it's worth noticing that the optimal design obtained by applying the RHS to the case B1 includes a 24-kW diesel generator; while in the same scenario, the LFS does not highlight the need for a backup source, with obvious consequences in terms of system reliability. This interesting result suggests that implementing the RHS during the sizing phase of the mini-grid can also change the cost-effectiveness of the diesel generator.

\begin{tabular}{|c|c|c|c|c|c|c|c|c|c|}
\hline \multirow{3}{*}{\multicolumn{2}{|c|}{$\begin{array}{c}\text { Operational strategy } \\
\text { Load curt. cost }(\$ / \mathrm{kWh}) \\
\text { Fuel delivery scenario }\end{array}$}} & \multicolumn{4}{|c|}{ Load following } & \multicolumn{4}{|c|}{ Rolling horizon } \\
\hline & & \multicolumn{2}{|c|}{0.5} & \multicolumn{2}{|c|}{1} & \multicolumn{2}{|c|}{0.5} & \multicolumn{2}{|c|}{1} \\
\hline & & A & B & A & B & A & B & A & B \\
\hline \multirow{3}{*}{ 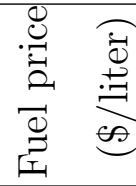 } & 0.8 & 95 & 100 & 122 & 129 & 46 & 46 & 56 & 57 \\
\hline & 1.2 & 0 & 0 & 113 & 114 & 24 & 23 & 52 & 57 \\
\hline & 1.6 & 0 & 0 & 100 & 99 & 0 & 0 & 43 & 49 \\
\hline
\end{tabular}

Table 3: Optimal size of the diesel generator $(\mathrm{kW})$.

\begin{tabular}{|c|c|c|c|c|c|c|c|c|c|}
\hline \multirow{2}{*}{\multicolumn{2}{|c|}{$\frac{\text { Operational strategy }}{\text { Load curt. cost }(\$ / \mathrm{kWh})}$}} & \multicolumn{4}{|c|}{ Load following } & \multicolumn{4}{|c|}{ Rolling horizon } \\
\hline & & \multicolumn{2}{|c|}{0.5} & \multicolumn{2}{|c|}{1} & \multicolumn{2}{|c|}{0.5} & \multicolumn{2}{|c|}{1} \\
\hline \multicolumn{2}{|c|}{ Fuel delivery scenario } & $\mathrm{A}$ & $\mathrm{B}$ & $\mathrm{A}$ & $\mathrm{B}$ & $\mathrm{A}$ & $\mathrm{B}$ & $\mathrm{A}$ & $\mathrm{B}$ \\
\hline \multirow{3}{*}{ 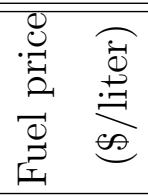 } & 0.8 & 2.3 & 4.0 & 3.4 & 5.5 & 3.2 & 5.2 & 3.9 & 8.0 \\
\hline & 1.2 & 0 & 0 & 3.2 & 4.2 & 1.5 & 2.2 & 3.6 & 4.5 \\
\hline & 1.6 & 0 & 0 & 2.9 & 3.1 & 0 & 0 & 2.3 & 4.0 \\
\hline
\end{tabular}

Table 4: Optimal size of the fuel tank (thousands of liters).

The optimal size of the fuel tank is reported in Table 4. First of all, these results confirm the findings of [15], where authors demonstrated that the optimal size of the tank increases with higher fuel price, cost of load curtailment and, obviously, the time required for fuel delivery. Present simulations also show what happens by changing the dispatching strategy of the mini-grid: with RHS the optimal size of the tank turns out to be higher than with LFS, even by 30\%. This is because RHS dispatches more frequently the diesel generator, whose fuel requirements increase by $24-35 \%$, depending on the analyzed scenario. 


\section{Conclusions}

Rural mini-grids suffer from scarce financing, so their sizing and operation at minimum costs are crucial. In this paper, we verified that sizing a hybrid mini-grid using a predictive operational strategy instead of implementing the traditional load-following approach enables coordinating the use of components, thus achieving promising savings in the Net Present Costs of the project. The proposed technique, which combines a probabilistic approach, an optimization tool and an intra-daily redispatching of resources, has proven to be effective in sizing an isolated system including the fuel tank and the fuel procurement, whose impact is typically neglected in the sizing process. The results have been enforced by using field-based data, collected from a mini-grid operated in Kenya.

The optimal sizes of photovoltaic plant, of the battery and of the converter obtained by the two strategies are similar, but the computational time required by the RHS is 500 times higher. This suggests that results of the LFS can be used to define the preliminary design of such components.

Conversely, the optimal sizes of the diesel generator and of the fuel tank are strictly dependent not only on the fuel price and on the cost of load curtailment, but also on the adopted dispatching strategy and, only for the fuel tank, also on the time expected for fuel delivery. In particular, in many economic scenarios the optimal sizes of the diesel generator and of the fuel tank are more than halved with RHS; in other cases, adopting the RHS procedure highlights the need for a backup diesel generator, whose necessity was not highlighted by the LFS. This study confirms that employing predictive strategies during the design phase of mini-grids can be a valid alternative or support to traditional sizing procedures.

Future research shall address and tackle the computational time of the RHS design stage. The utilization of other techniques (e.g. robust or stochastic optimization) that also consider the future uncertainty of the load growth shall be compared to the RHS and the LFS for sizing the PV, the batteries and the diesel generator. The uncertainty regarding the long-term evolution of the load will be treated as well. Finally, the sizing and operating procedures developed in this study will be tested on real mini-grids, thus assessing the complexity of implementation of predictive strategies in substitution or support of the traditional load-following approach. 


\section{References}

[1] IEA, World Energy Outlook 2015, Tech. rep., IEA (2015).

[2] UN, Energy for a Sustainable Future, Tech. rep., UN Secretary-General's Advisory Group on Energy and Climate Change (2010).

[3] Y. Borofsky, I. Perez-Arriaga, R. Stoner, A model for better electrification planning, ABB Review (2) (2017) 23-27.

[4] D. Ellman, The Reference Electrification Model : A Computer Model for Planning Rural Electricity Access, Ph.D. thesis (2015).

[5] IRENA, Innovation Outlook: Renewable Mini-Grids, Tech. rep., IRENA (2016).

[6] C. T. Gaunt, E. Namanya, R. Herman, Voltage modelling of LV feeders with dispersed generation: Limits of penetration of randomly connected photovoltaic generation, Elect. Power Syst. Research 143 (2017) 1-6.

[7] J. Hazelton, A. Bruce, I. MacGill, A review of the potential benefits and risks of photovoltaic hybrid mini-grid systems, Renewable Energy 67 (2014) 222-229.

[8] D. Fioriti, R. Giglioli, D. Poli, G. Lutzemberger, A. Vanni, P. Salza, Optimal sizing of a hybrid mini-grid considering the fuel procurement and a rolling horizon system operation, in: EEEIC, 2017.

[9] S. Mazzola, C. Vergara, M. Astolfi, V. Li, I. Perez-Arriaga, E. Macchi, Assessing the value of forecast-based dispatch in the operation of off-grid rural microgrids, Renewable Energy 108.

[10] R. Siddaiah, R. P. Saini, A review on planning, configurations, modeling and optimization techniques of hybrid renewable energy systems for off grid applications, Renewable and Sustainable Energy Reviews 58 (2016) 376-396.

[11] O. O. Amusat, P. R. Shearing, E. S. Fraga, Optimal design of hybrid energy systems incorporating stochastic renewable resources fluctuations, Journal of Energy Storage 15 (2018) 379-399. 
[12] M. Sharafi, T. Y. ElMekkawy, Stochastic optimization of hybrid renewable energy systems using sampling average method, Renewable and Sustainable Energy Reviews 52 (2015) 1668-1679.

[13] N. Sharma, Varun, Siddhartha, Stochastic techniques used for optimization in solar systems: A review, Renewable and Sustainable Energy Reviews 16 (3) (2012) 1399-1411.

[14] P. Prakash, D. K. Khatod, Optimal sizing and siting techniques for distributed generation in distribution systems: A review, Renewable and Sustainable Energy Reviews 57 (2016) 111-130.

[15] D. Fioriti, R. Giglioli, D. Poli, G. Lutzemberger, A. Vanni, P. Salza, Optimal sizing of a mini-grid in developing countries, taking into account the operation of an electrochemical storage and a fuel tank, in: International conference on clean electrical power (ICCEP), 2017.

[16] M. D. Al-falahi, S. D. Jayasinghe, H. Enshaei, A review on recent size optimization methodologies for standalone solar and wind hybrid renewable energy system, Energy Conv. and Manag. 143 (2017) 252-274.

[17] C. Brivio, M. Moncecchi, S. Mandelli, M. Merlo, A novel software package for the robust design of off-grid power systems, J. of Cleaner Production 166 (2017) 668-679.

[18] S. Mandelli, C. Brivio, E. Colombo, M. Merlo, Effect of load profile uncertainty on the optimum sizing of off-grid PV systems for rural electrification, Sustain. Energy Technol. and Assess. 18 (2016) 34-47.

[19] R. Luna-Rubio, M. Trejo-Perea, D. Vargas-Vázquez, G. J. Ríos-Moreno, Optimal sizing of renewable hybrids energy systems: A review of methodologies, Solar Energy 86 (4) (2012) 1077-1088.

[20] Z. Liu, F. Wen, G. Ledwich, Optimal siting and sizing of distributed generators in distribution systems considering uncertainties, IEEE Transactions on Power Delivery 26 (4) (2011) 2541-2551.

[21] S. Xia, K. Chan, X. Luo, S. Bu, Z. Ding, B. Zhou, Optimal sizing of energy storage system and its cost-benefit analysis for power grid planning with intermittent wind generation, Renewable Energy 122 (2018) $472-486$. 
[22] A. Maleki, M. G. Khajeh, M. Ameri, Optimal sizing of a grid independent hybrid renewable energy system incorporating resource uncertainty, and load uncertainty, Int. J. of Electr. Power and Energy Syst. 83.

[23] K. H. Chang, G. Lin, Optimal design of hybrid renewable energy systems using simulation optimization, Simulation Modelling Practice and Theory 52 (2015) 40-51.

[24] Homer Energy, HOMER Pro Version 3.7 User Manual, Tech. Rep. August, HOMER (2016).

[25] S. Mazzola, M. Astolfi, E. Macchi, The potential role of solid biomass for rural electrification: A techno economic analysis for a hybrid microgrid in India, Applied Energy 169 (2016) 370-383.

[26] S. Murphy, The construction of a modified Typical Meteorological Year for photovoltaic modeling in India, Renew. Energy 111 (2017) 447-454.

[27] T. R. Ayodele, A. S. O. Ogunjuyigbe, C. G. Monyei, On the global solar radiation prediction methods, J. of Renew. and Sustain. Energy 8 (2).

[28] V. A. Graham, K. G. T. Hollands, T. E. Unny, A Time Series Model for Kt with Application to Global Synthetic Weather Generation, Solar Energy 40 (2) (1988) 83-92.

[29] V. A. Graham, K. G. T. Hollands, A method to generate synthetic hourly solar radiation globally, Solar Energy 44 (6) (1990) 333-341.

[30] S. M. Hakimi, S. M. Moghaddas-Tafreshi, Optimal sizing of a standalone hybrid power system via particle swarm optimization for Kahnouj area in south-east of Iran, Renewable Energy 34 (7) (2009) 1855-1862.

[31] Z. Lavan, J. Thompson, Experimental study of thermally stratified hot water storage tanks, Solar Energy 19 (5) (1977) 519-524.

[32] L.-H. Shih, Planning of fuel coal imports using a mixed integer programming method, Int. J. of Production Economics 51 (3) (1997) 243-249.

[33] C. D. Barley, C. B. Winn, Optimal dispatch strategy in remote hybrid power systems, Solar Energy 58 (4-6) (1996) 165-179. 
[34] L. Olatomiwa, S. Mekhilef, M. S. Ismail, M. Moghavvemi, Energy management strategies in hybrid renewable energy systems: A review, Renewable and Sustainable Energy Reviews 62 (2016) 821-835.

[35] S. Barsali, R. Giglioli, M. Giuntoli, G. Lutzemberger, D. Poli, Control strategies and real time operation of storage systems integrated with MV photovoltaic plants, in: 2015 IEEE 15th International Conference on Environment and Electrical Engineering, EEEIC 2015 - Conference Proceedings, Institute of Electrical and Electronics Engineers Inc., 2015, pp. 243-248.

[36] D. Fioriti, R. Giglioli, D. Poli, Short-term operation of a hybrid minigrid under load and renewable production uncertainty, in: Global Humanitarian Technology Conference (GHTC), 2016 IEEE, 2016, pp. 436-443.

[37] L. Wu, M. Shahidehpour, Z. Li, Comparison of scenario-based and interval optimization approaches to stochastic SCUC, IEEE Transactions on Power Systems 27 (2) (2012) 913-921.

[38] A. Fathy, H. Rezk, Parameter estimation of photovoltaic system using imperialist competitive algorithm, Renew. Energy 111 (2017) 307-320.

[39] A. Micangeli, R. Del Citto, I. Kiva, S. Santori, V. Gambino, J. Kiplagat, D. Viganò, D. Fioriti, D. Poli, Energy Production Analysis and Optimization of Mini-Grid in Remote Areas: The Case Study of Habaswein, Kenya, Energies 10 (12) (2017) 2041.

[40] A. J. Kleywegt, A. Shapiro, T. Homem-de Mello, The Sample Average Approximation Method for Stochastic Discrete Optimization, SIAM Journal on Optimization 12 (2) (2002) 479-502.

[41] G. Giannuzzi, F. Bassi, M. Giuntoli, P. Pelacchi, D. Poli, Mechanical Behaviour of Multi-Span Overhead Transmission Lines Under Dynamic Thermal Stress of Conductors Due to Power Flow and Weather Conditions, International Review on Modelling and Simulations (IREMOS) 6 (4) (2013) 1112-1122.

[42] M. Giuntoli, P. Pelacchi, D. Poli, On the use of simplified reactive power flow equations for purposes of fast reliability assessment, in: Eurocon 2013, IEEE, 2013, pp. 992-997. 
[43] Republic of Kenya, Wajir First County Integrated Development Plan, Tech. rep., Republic of Kenya (2013).

URL https://cog.go.ke/images/stories/CIDPs/Wajir.pdf

[44] M. Woodhouse, R. Jones-Albertus, D. Feldman, R. Fu, K. Horowitz, D. Chung, D. Jordan, S. Kurtz, On the Path to SunShot: The Role of Advancements in Solar Photovoltaic Efficiency, Reliability, and Costs, Tech. Rep. May (2016).

[45] A. Sangwongwanich, Y. Yang, D. Sera, F. Blaabjerg, Lifetime evaluation of PV inverters considering panel degradation rates and installation sites, IEEE Transactions on Power Electronics 33 (2) (2018) 1225-1236.

[46] P. Mohanty, T. Muneer, E. J. Gago, Y. Kotak, Solar radiation fundamentals and PV system components, in: Solar Photovoltaic System Applications, Vol. 196, Springer Verlag, 2016, pp. 7-47.

[47] IRENA, Renewable Power Generation Costs in 2017, Tech. rep. (2018).

[48] Bloomberg, New Energy Outlook 2017, Tech. rep., Bloomberg (2017).

[49] R. Fu, D. Chung, T. Lowder, D. Feldman, K. Ardani, R. Fu, D. Chung, T. Lowder, D. Feldman, K. Ardani, U.S. Solar Photovoltaic System Cost Benchmark: Q1 2017, Tech. Rep. September (2017).

[50] Generator Sales, Diesel generators (2017).

URL https://www.centralmainediesel.com/diesel-generators. asp

[51] Fuel Tank Shop, Diesel tank (2017).

URL https://www.fueltankshop.co.uk/diesel/c108 